\title{
Two-dimensional slices of nonpseudoconvex domains with rough boundary
}

\author{
Egmont Porten
}

Received: 19 March 2013 / Accepted: 2 February 2014 / Published online: 10 June 2014

(C) Springer-Verlag Berlin Heidelberg 2014

\begin{abstract}
For a domain $D \subset \mathbb{C}^{n}, n \geq 3$, the set $E$ is defined as the set of all points $z \in \mathbb{C}^{n}$ for which the intersection of $D$ with every complex 2-plane through $z$ is pseudoconvex. For $D$ nonpseudoconvex, it is shown that $E$ is contained in an affine subspace of codimension 2. This results solves a problem raised by Nikolov and Pflug.
\end{abstract}

Keywords Pseudoconvexity - Sections of nonpseudoconvex domains · Continuity principle

Mathematics Subject Classification $\quad 32 \mathrm{~T} 05 \cdot 32 \mathrm{D} 20 \cdot 32 \mathrm{D} 26 \cdot 32 \mathrm{~A} 40$

\section{Introduction}

It was observed by Hitotumatu [2], see also [5], that a domain $D \subset \mathbb{C}^{n}, n \geq 3$, is pseudoconvex iff its intersections with all 2-dimensional affine complex planes $P$ are pseudoconvex open sets. On the other hand, the family of planes passing through a single point does not suffice to detect nonpseudoconvexity. For a nonpseudoconvex domain $D \subset \mathbb{C}^{n}$, we call $z \in \mathbb{C}^{n}$ exceptional if the intersection $P \cap D$ is pseudoconvex for every 2-plane $P$ passing through $z$ (the empty set is considered as pseudoconvex). We denote the set of exceptional points by $E$. As an example for a large $E$, we may consider a pseudoconvex $G \subset \mathbb{C}^{3}$ and a line $\ell \subset \mathbb{C}^{3}$ with $\ell \cap G \neq \emptyset$, in which case the points in $\ell \backslash G$ are exceptional for $D=G \backslash \ell$, see [7]. In general the set $E$ cannot be too large: In [7] it was proved that the set $E$ of exceptional points is always contained in an affine complex hyperplane of $\mathbb{C}^{n}$.

For domains with higher boundary regularity, a finer study of $E$ is given in [6]. There it is shown for certain domains in $\mathbb{C}^{3}$ that $E$ is contained in a line. Moreover the question is raised whether this holds for every domain in $\mathbb{C}^{3}[6$, Remark 3 (1)]. Our main result answers the question in the affirmative.

E. Porten $(\bowtie)$

Department for Science Education and Mathematics, Mid Sweden University, Sundsvall 85170, Sweden e-mail: Egmont.Porten@miun.se 
Theorem 1.1 Let $D \subset \mathbb{C}^{n}, n \geq 3$, be a nonpseudoconvex domain. Then the set $E$ of exceptional points is contained in an affine complex subspace of codimension two.

The above-mentioned example shows that the theorem is sharp. If one assumes that $\partial D$ is more regular, stronger conclusions are possible [6]: For example, $E$ is empty for a nonpseudoconvex $D$ with $\mathcal{C}^{2}$-boundary.

\section{Proof of the main result}

Assume that $E$ contains $n$ points $p_{0}, p_{1}, \ldots, p_{n-1}$ which do not lie in a common affine subspace of dimension $n-2$. The theorem will be proved as soon as we have deduced that $D$ is pseudoconvex. After an affine coordinate change, we can assume that $p_{0}, p_{1}, \ldots, p_{n-1}$ are contained in $H=\left\{z_{n}=0\right\}$. We start by

Lemma 2.1 The complement $D_{1}=D \backslash H$ is a pseudoconvex domain.

Proof of Lemma 2.1 Observe that $p_{0}, p_{1}, \ldots, p_{n-1}$ are also exceptional points of $D_{1}$. Indeed, for a plane $P$ through $p_{j}$ which is not contained in $H$ the intersection $P \cap D_{1}$ equals $P \cap D$ minus a complex line, which is pseudoconvex or empty.

Assume that $D_{1}$ is not pseudoconvex. As pointed out in [7, Proposition 3], there is a point $q \in \partial D_{1}$, affine coordinates $w_{1}=u_{1}+i v_{1}, \ldots, w_{n}=u_{n}+i v_{n}$ centered at $q$ and constants $\eta>0, c<1$, such that

$$
G=\left\{w \in \mathbb{C}^{n}: u_{n}<u_{1}^{2}-c v_{1}^{2}-\left|w_{2}\right|^{2}-\cdots-\left|w_{n-1}\right|^{2}-v_{n}^{2},|w|<\eta\right\}
$$

is contained in $D_{1}$. Now $c<1$ means that $\partial G \cap\left\{w_{2}=\cdots=w_{n-1}=0\right\}$ is strictly pseudoconcave at $q$ in the $\left(w_{1}, w_{n}\right)$-plane. We claim that $q \notin H$. Otherwise the hyperplane $H$, which lies in the complement of $G \subset D_{1}$, would coincide with the complex tangent space $T_{q}=T_{q}^{c} \partial G$ of $\partial G$ at $q$ (viewed as affine hyperplane of $\left.\mathbb{C}^{n}\right)$. Thus $T_{q} \cap\left\{w_{2}=\cdots=\right.$ $\left.w_{n-1}=0\right\}$ would touch $G \cap\left\{w_{2}=\cdots=w_{n-1}=0\right\}$ from outside, in contradiction to strict pseudoconcavity.

Next we claim that the exceptional points of $D_{1}$ are contained in $T_{q}$. The reason is essentially contained in [7, Proposition 13] and its proof: For $q^{\prime} \notin T_{q}$, we consider the plane $P=q+\operatorname{span}_{\mathbb{C}}\left\{q-q^{\prime}, e_{1}\right\}$, where $e_{1}=(1,0, \ldots, 0)$ is taken in the coordinates $w_{1}, \ldots, w_{n}$. Then $q$ is a strictly pseudoconcave point of $G \cap P$, meaning that $D_{1} \cap P$ cannot be pseudoconvex either.

From the claim we get $p_{0}, p_{1}, \ldots, p_{n-1} \in T_{q}$, which is impossible since $T_{q} \neq H$.

To study the geometry of $D$ along $H$, we first consider another auxiliary domain $D_{2} \supset D_{1}$, which may be strictly larger than $D$.

Lemma 2.2 The set $D_{2}$ obtained from $D_{1}$ by adding every point $z \in H$ which has an ambient neighborhood $U$ satisfying $U \backslash H \subset D_{1}$, is a pseudoconvex domain.

Lemma 2.2 is a consequence of a classical result of Grauert and Remmert [1, Satz 4], since $D_{2}$ is pseudoconvex near points on $\partial D_{2} \backslash H$ and $\partial D_{2} \cap H$ is a thin set without removable points (in the terminology of [1]). For the reader's convenience, we provide a short proof using Hörmander's $L^{2}$ theory [3] and an idea from [8]. The final version of the argument was communicated by P. Pflug. 
Proof of Lemma 2.2 Let $G \subset \mathbb{C}^{n}$ be a domain. We call a pair $(U, V)$ of domains in $\mathbb{C}^{n}$ an extension pair for $G$ if (i) $V \not \subset G$, (ii) $V \cap G \neq \emptyset$, (iii) $U$ is a connected component of $V \cap G$, and (iv) for every $f \in \mathcal{O}(G)$, there is $\tilde{f} \in \mathcal{O}(V)$ which coincides with $f$ on $U$. By classical Cartan-Thullen theory (see [2,4]), $G$ is pseudoconvex if and only if it does not admit any extension pair.

Obviously $D_{2}$ is a domain. Let us assume that $D_{2}$ is not pseudoconvex, implying the existence of an extension pair $(U, V)$ for $D_{2}$. Note that $(U \backslash H, V \backslash H)$ satisfies the properties (i-iii) for $G=D_{1}$. Indeed, (ii) and (iii) hold since we have subtracted a subspace of real codimension 2. As for (i), $(V \backslash H) \subset D_{1}$ would imply $V \cap H \subset D_{2}$ (by the definition of $D_{2}$ ) and yield the contradiction $V \subset D_{2}$.

We will exploit a structure theorem on holomorphic functions with polynomial growth. Let $\delta_{D_{1}}(z)$ be the euclidean distance between $z \in D_{1}$ and $\partial D_{1}$. By [4, Proposition 2.5.5] and Lemma 2.1, $D_{1}$ is also a domain of holomorphy with respect to the Banach space

$$
\mathcal{O}^{(2 n+1)}\left(D_{1}\right)=\left\{f \in \mathcal{O}(X) \mid \delta_{D_{1}}^{(2 n+1)} f \text { is bounded onD } 1\right\},
$$

meaning that for every point in $w \in \partial D_{1}$, there is a function in $\mathcal{O}^{(2 n+1)}\left(D_{1}\right)$ without holomorphic extension across $w$.

Since $(U \backslash H) \varsubsetneqq(V \backslash H)$ there is a point $z_{0} \in \partial(U \backslash H) \cap(V \backslash H)$. From (iii) we get $z_{0} \in \partial D_{1}$. The structure theorem yields $f \in \mathcal{O}^{(2 n+1)}\left(D_{1}\right)$ without extension through $z_{0}$. Riemann's theorem on removable singularities implies that $g=z^{2 n+1} f$ is holomorphic on $D_{2}$ and therefore $\left.g\right|_{U}$ admits an extension $\tilde{g} \in \mathcal{O}(V)$. But this means that $\left.f\right|_{U}$ has an extension $\tilde{g} / z^{2 n+1} \in \mathcal{O}(V \backslash H)$, a contradiction to the choice of $z_{0}$ and $f$. The proof of Lemma 2.2 is complete.

By construction we have $D \subset D_{2}$ and $D \backslash H=D_{1}=D_{2} \backslash H$. We continue by studying $\widehat{D}=D \cap H, \widehat{D}_{2}=D_{2} \cap H$. These are of course open subsets of $H$ satisfying $\widehat{D} \subset \widehat{D}_{2}$.

Lemma 2.3 The region $\widehat{D}$ is a union of connected components of $\widehat{D}_{2}$.

Proof of Lemma 2.3 The proof relies on a propagation property. Assume that the statement fails, i.e. that there is a connected component $W$ of $\widehat{D}_{2}$ with $\emptyset \neq W \cap \widehat{D} \varsubsetneqq W$. Using that connected open sets are path connected, we find a continuous curve $\gamma:[0,1] \rightarrow W$ satisfying $\gamma(0) \in \widehat{D}_{2} \backslash \widehat{D}$ and $\gamma((0,1]) \subset \widehat{D}$. The crucial step is to replace $\gamma$ by a well-chosen straight segment to which we can apply propagation arguments. We claim:

There is a compact straight real segment I $\subset$ H contained in a complex line through one of the points $p_{0}, p_{1}, \ldots, p_{n-1}$ such that one extremal point of $I$ is contained in $\widehat{D}_{2} \backslash \widehat{D}$ and all other points in $\widehat{D}$.

In preparation we construct a suitable parametrization of $H$ near $w=\gamma(0)$. For two points $p$ and $q$ we denote by $\overrightarrow{p q}$ the vector pointing from $p$ to $q$ and by $[p, q]$ the straight (real) segment linking the points. Observe that there are $n-1 \mathbb{C}$-independent vectors among $v_{j}=\overrightarrow{w p}_{j}, j=0, \ldots, n-1$. In fact, the $v_{j}$ span $H$, and we can select a basis.

Suppose $v_{1}, \ldots, v_{n-1}$ independent. Set

$$
F_{1}\left(\zeta_{1}\right)=w+\zeta_{1} v_{1}
$$

and define inductively for $k=2, \ldots, n-1$ the mappings

$$
F_{k}\left(\zeta_{1}, \ldots, \zeta_{k}\right)=F_{k-1}\left(\zeta_{1}, \ldots, \zeta_{k-1}\right)+\zeta_{k} \overrightarrow{F_{k-1}\left(\zeta_{1}, \ldots, \zeta_{k-1}\right) p_{k}}
$$

Taking the differential at $\zeta=0$, we see that the mapping $F=F_{n-1}$ is a biholomorphism of the $(n-1)$-dimensional polydisc $\left\{\max \left|\zeta_{j}\right|<\eta\right\}$ onto a neighborhood of $w$ in $H$, if $\eta>0$ is small. It is crucial that for $\zeta_{1}, \ldots, \zeta_{\ell}$ fixed, the image of 


$$
\lambda \mapsto F\left(\zeta_{1}, \ldots, \zeta_{\ell}, \lambda, 0, \ldots, 0\right)
$$

is contained in a line through $p_{\ell}$.

After shortening $\gamma$, we may assume that $\gamma$ is contained in

$$
U=F\left(\left\{\max \left|\zeta_{j}\right|<\eta\right\}\right) \subset W .
$$

We link $w=\gamma(0)$ and $\gamma(1)=F\left(\hat{\zeta}_{1}, \ldots, \hat{\zeta}_{n-1}\right)$ by the concatenation of the straight segments

$$
\begin{gathered}
{\left[w, F\left(\hat{\zeta}_{1}, 0, \ldots, 0\right)\right],\left[F\left(\hat{\zeta}_{1}, 0, \ldots, 0\right), F\left(\hat{\zeta}_{1}, \hat{\zeta}_{2}, 0, \ldots, 0\right)\right], \ldots,} \\
{\left[F\left(\hat{\zeta}_{1}, \ldots, \hat{\zeta}_{n-2}, 0\right), F\left(\hat{\zeta}_{1}, \ldots, \hat{\zeta}_{n-1}\right)\right] .}
\end{gathered}
$$

By construction each of these segments lies on a complex line through one of the $p_{j}$. Since there is a last point in $\widehat{D}_{2} \backslash \widehat{D}$ on the concatenation, we obtain a segment $I$ as required by taking an appropriate subsegment. The claim is proved.

By construction $I$ lies on a complex line $L$ passing through one of the points $p_{0}, \ldots, p_{n-1}$. Hence the intersection of $D$ with the plane

$$
Q=\left\{\left(z_{1}, \ldots, z_{n}\right) \in \mathbb{C}^{n}:\left(z_{1}, \ldots, z_{n-1}, 0\right) \in L\right\}
$$

with $D$ is pseudoconvex. On the other hand, the one-parameter family of flat complex discs

$$
\Delta_{\left(z_{1}, \ldots, z_{n-1}\right)}=\left\{\left(z_{1}, \ldots, z_{n-1}\right\} \times\left\{\left|z_{n}\right| \leq \varepsilon\right\},\left(z_{1}, \ldots, z_{n-1}, 0\right) \in I,\right.
$$

violates the continuity principle (with respect to $D \cap Q$ ) if $\varepsilon>0$ is small enough. This contradiction completes the proof of Lemma 2.3.

Since $D$ is obtained by taking out of $D_{2}$ a collection of properly embedded complex hypersurfaces, it is pseudoconvex. The proof of Theorem 1.1 is complete.

The main theorem and its proof allow a natural generalization to Riemann domains $\pi$ : $X \rightarrow \mathbb{C}^{n}, n \geq 3$. For an affine complex 2-plane $P$ we consider $P_{X}=\pi^{-1}(P)$. For a nonpseudoconvex $X$, we call $z \in \mathbb{C}^{n}$ exceptional if the intersection $P_{X}$ is pseudoconvex for every 2-plane $P$ passing through $z$, and denote by $E$ the set of exceptional points. We obtain

Corollary 2.4 If $\pi: X \rightarrow \mathbb{C}^{n}, n \geq 3$, is not pseudoconvex, then $E$ is contained in a complex affine subspace of $\mathbb{C}^{n}$ of codimension two.

Acknowledgments The author would like to thank Nikolai Nikolov and Peter Pflug for valuable remarks. In particular, I am grateful to Peter Pflug for pointing out a confusion in the original proof of Lemma 2.2, and for providing an elegant argument, which both rectifies and shortens the original reasoning.

\section{References}

1. Grauert, H., Remmert, R.: Konvexität in der komplexen analysis. Comment. Math. Helvetici 31, 152-183 (1956)

2. Hitotumatu, S.: On some conjectures concerning pseudo-convex domains. J. Math. Soc. Jpn. 6, 177-195 (1954)

3. Hörmander, L.: An Introduction to Complex Analysis in Several Variables. North-Holland, Amsterdam (1990)

4. Jarnicki, M., Pflug, P.: Extension of holomorphic functions. De Gruyter Expositions in Mathematics, vol. 34. Walter de Gruyter, Berlin (2000)

5. Jacobson, R.: Pseudoconvexity is a two-dimensional phenomenon. Preprint arXiv:0907.1304v1

6. Nikolov, N., Pflug, P.: Two-dimensional slices on non-pseudoconvex open sets. Math. Z. 272, 381-388 (2012) 
7. Nikolov, N., Thomas, P.J.: Rigid characterizations of pseudoconvex domains. Indiana Univ. Math. J. 61, 1313-1323 (2012)

8. Porten, E.: On the Hartogs-phenomenon and extension of analytic hypersurfaces in non-separated Riemann domains. Complex Var. 47, 325-332 (2002) 